\title{
Anisotropic Quantum Well Electro-Optics in Few-Layer Black Phosphorus
}

Michelle C. Sherrott, William S. Whitney, Deep Jariwala, Souvik Biswas, Cora Went, Joeson Wong, George R. Rossman, and Harry A Atwater

Nano Lett., Just Accepted Manuscript • Publication Date (Web): 07 Dec 2018

Downloaded from http://pubs.acs.org on December 7, 2018

\section{Just Accepted}

"Just Accepted" manuscripts have been peer-reviewed and accepted for publication. They are posted online prior to technical editing, formatting for publication and author proofing. The American Chemical Society provides "Just Accepted" as a service to the research community to expedite the dissemination of scientific material as soon as possible after acceptance. "Just Accepted" manuscripts appear in full in PDF format accompanied by an HTML abstract. "Just Accepted" manuscripts have been fully peer reviewed, but should not be considered the official version of record. They are citable by the Digital Object Identifier (DOI®). "Just Accepted" is an optional service offered to authors. Therefore, the "Just Accepted" Web site may not include all articles that will be published in the journal. After a manuscript is technically edited and formatted, it will be removed from the "Just Accepted" Web site and published as an ASAP article. Note that technical editing may introduce minor changes to the manuscript text and/or graphics which could affect content, and all legal disclaimers and ethical guidelines that apply to the journal pertain. ACS cannot be held responsible for errors or consequences arising from the use of information contained in these "Just Accepted" manuscripts. 


\title{
Anisotropic Quantum Well Electro-Optics in Few-Layer Black Phosphorus
}

Michelle C. Sherrott ${ }^{1,2 \ddagger}$, William S. Whitney ${ }^{3 \ddagger}$, Deep Jariwala ${ }^{1,2, \wedge}$, Souvik Biswas ${ }^{1}$, Cora M. Went $^{2,3}$, Joeson Wong ${ }^{1}$, George R. Rossman ${ }^{54}$, Harry A. Atwater*1,2,5

1. Thomas J. Watson Laboratory of Applied Physics, California Institute of Technology, Pasadena, CA 91125, USA

2. Resnick Sustainability Institute, California Institute of Technology, Pasadena, CA 91125, USA

3. Department of Physics, California Institute of Technology, Pasadena, CA 91125, USA

4. Division of Geological and Planetary Sciences, California Institute of Technology, Pasadena, CA 91125, USA

5. Joint Center for Artificial Photosynthesis, California Institute of Technology, Pasadena, CA 91125, USA

₹ Equal contributors

^ Current affiliation, Department of Electrical and Systems Engineering, University of Pennsylvania, Philadelphia, PA, 19104, USA

*Corresponding author: Harry A. Atwater (haa@caltech.edu)

\begin{abstract}
The incorporation of electrically tunable materials into photonic structures such as waveguides and metasurfaces enables dynamic, electrical control of light propagation at the nanoscale. Few-layer black phosphorus is a promising material for these applications due to its in-plane anisotropic, quantum well band structure, with a direct band gap that can be tuned from $0.3 \mathrm{eV}$ to $2 \mathrm{eV}$ with number of layers and subbands that manifest as additional optical transitions across a wide range of energies. In this work, we report an experimental investigation of three different, anisotropic electro-optic mechanisms that allow electrical control of the complex refractive index in few-layer black phosphorus from the mid-infrared to the visible: Pauliblocking of intersubband optical transitions (the Burstein-Moss effect); the quantum-confined Stark effect; and the modification of quantum well selection rules by a symmetry-breaking, applied electric field. These effects generate near-unity tuning of the BP oscillator strength for some material thicknesses and photon energies, along a single in-plane crystal axis, transforming absorption from highly anisotropic to nearly isotropic. Lastly, the anisotropy of these electro-optical phenomena results in dynamic control of linear dichroism and


birefringence, a promising concept for active control of the complex polarization state of light, or propagation direction of surface waves.

Keywords: Electro-optic tunability, black phosphorus, broadband, anisotropy, van der Waals materials

Dynamic control of the near and far-field propagation of light is critical for next-generation optoelectronic devices. Ultra-thin, layered materials are promising building blocks for this functionality, as they are easily incorporated into atom-scale structures, and their optical properties can be changed dramatically under applied electric fields ${ }^{1,2}$. Few-layer black phosphorus (BP) is particularly compelling due to its high electronic mobility, in-plane anisotropy, and thickness-tunable quantum well band structure, with a direct band gap that varies from $0.3 \mathrm{eV}$ in bulk to $2 \mathrm{eV}$ for monolayers ${ }^{3,4}$. Recent work using electrostatic gating and potassium ions has further shown that the electronic band gap of BP may be tuned by an electric field ${ }^{5-8}$. These unique attributes have already enabled the realization of novel and highperformance optoelectronic devices, including waveguide-integrated photodetectors ${ }^{9-13}$.

One of the most unusual features of BP is its large in-plane structural anisotropy, which generates to a polarization-dependent optical response ${ }^{14-16}$ as well as mechanical ${ }^{17}$, thermal ${ }^{18}$, and electrical transport characteristics ${ }^{19,20}$ that vary with in-plane crystallographic orientation ${ }^{21}$. This optical anisotropy corresponds to a large, broadband birefringence ${ }^{22}$, wherein the distinct optical index of refraction along each axis leads to a phase delay between polarization states of light. Moreover, mirror-symmetry in the $\mathrm{x}-\mathrm{z}$ plane forbids intersubband optical transitions along the zigzag axis, and as a result, BP exhibits significant linear dichroism, wherein the material absorption depends strongly on the polarization state of exciting light ${ }^{15,23}$. An additional constraint on optical absorption corresponds to the naturally occurring quantum well nature of the BP band structure, wherein optical transitions are only allowed between subbands of opposite parities, because photons carry parity -1 . For low energy transitions, this corresponds 
to transitions between bands of equal quantum number (ie: $v_{1}$ to $c_{1}, v_{2}$ to $c_{2}$ ), described in depth in Ref [19].

In this work, we use multiple field-effect device configurations to isolate and characterize three distinct, anisotropic electro-optic effects that allow significant control of the complex refractive index in BP. These effects are Pauli-blocking of intersubband optical transitions, also known as a Burstein-Moss or band-filling effect; the quantum confined Stark effect; and, modification of quantum well selection rules by a symmetry-breaking electric field. The resulting response approaches near-unity tunability of the BP oscillator strength for some BP thicknesses and photon energies. Further, this tuning is only along one in-plane crystal axis (the armchair direction). As a result, we are able to electrically control dichroism and birefringence in BP. In some cases, we observe tuning of the black phosphorus optical response from highly anisotropic to nearly isotropic. We observe this anisotropic tunability from the visible to midinfrared (mid-IR) spectral regimes, behavior not seen in traditional electro-optic materials such as graphene ${ }^{24}$, transparent conducting oxides ${ }^{25,26}$, silicon ${ }^{27}$, and quantum wells ${ }^{28}$. This opens up the possibility of realizing novel photonic structures in which linear dichroism in the van der Waals plane can be continuously tuned with low power consumption, because the switching is electrostatic in nature. By controlling optical losses in the propagation plane, for example, efficient in-plane beam steering of surface plasmon polaritons or other guided modes is enabled. Moreover, an electrically tunable polarizer could be realized by modulating the polarization state of light absorbed in a resonant structure containing BP. Because this tunability is strongest at infrared wavelengths, it could also enable control of the polarization state of thermal radiation ${ }^{29-31}$.

In order to probe and distinguish the electro-optical tuning mechanisms evident in few-layer BP, we used a combination of gating schemes wherein the BP either floats electrically in an applied field or is contacted, as shown in Fig. 1a and described further in the Methods section. We note that for all experiments, the samples are exfoliated on an oxide surface and encapsulated in $\mathrm{Al}_{2} \mathrm{O}_{3}$; therefore, while strain effects are known to influence the band gap of 2D 
materials ${ }^{32-34}$, including few-layer $\mathrm{BP}$, these are identical for all measurements. Polarizationdependent optical measurements are taken aligned to the crystal axes, in order to probe the structural anisotropy shown in Fig. 1b. This enables us to isolate the contribution of chargecarrier density effects - i.e. a Burstein-Moss shift - and external field-effects - i.e.: the quantum-confined Stark effect and control of forbidden transitions in the infrared - to the tunability of linear dichroism, qualitatively illustrated in Figures $1 \mathrm{c}$ and $1 d^{35-38}$. In the anisotropic Burstein-Moss (BM) shift, the optical band gap of the material is changed as a result of band filling and the consequent Pauli-blocking of intersubband transitions. As the carrier concentration of the sample is changed, the Fermi level moves into (out of) the conduction or valence band, resulting in a decrease (increase) of absorptivity due to the disallowing (allowing) of optical transitions ${ }^{39,40}$. Because intersubband optical transitions are only allowed along the armchair axis of $\mathrm{BP}^{15,21,41}$, this tunability occurs only for light polarized along this axis. In the quantum-confined Stark Effect, the presence of a strong electric field results in the leaking of electron and hole wave functions into the band gap as Airy functions, red-shifting the intersubband transitions energies ${ }^{42}$. In quantum well structures, this red-shifting is manifested for multiple subbands, and therefore can be observed over a wide range of energies above the band gap. To assess the gate-tunable anisotropy of the optical response of $\mathrm{BP}$, the armchair and zigzag axes, illustrated in Fig. 1b, of the samples considered are identified by a combination of cross-polarized visible microscopy, described in the Supporting Information S1, and either polarization-dependent Raman spectroscopy or infrared measurements. We perform polarization-dependent Raman spectroscopy with the $532 \mathrm{~nm}$ excitation laser polarized parallel to one of the two crystal axes as determined by cross-polarized visible microscopy. The armchair and zigzag axes cannot be distinguished from the intensity of any of the Raman peaks alone, as the polarization behavior of each peak depends strongly on the excitation wavelength and the flake thickness ${ }^{43}$. However, for $532 \mathrm{~nm}$ excitation and all thicknesses, the ratio of the intensities of the $A^{2} g$ and the $A^{1} g$ Raman peaks is higher for the armchair than the zigzag axis, so this ratio remains a robust way to distinguish between the axes ${ }^{43,44}$. Representative Raman spectra are presented for the visible frequency sample on $\mathrm{SrTiO}_{3}$ in Figure 1e. 
a

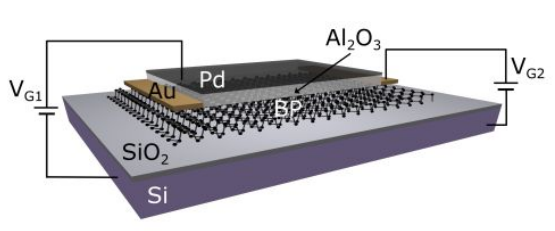

C

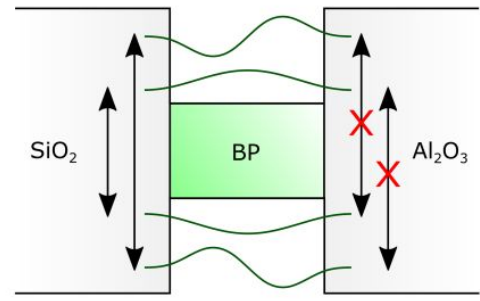

b

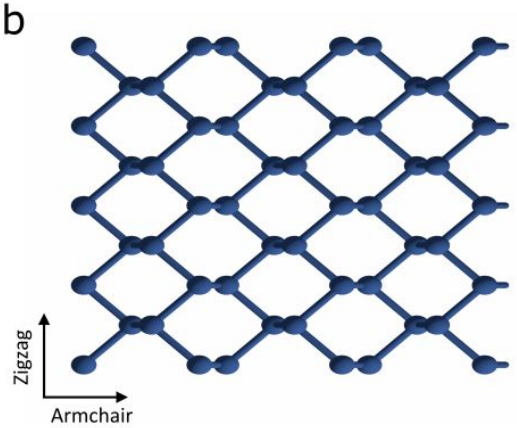

d

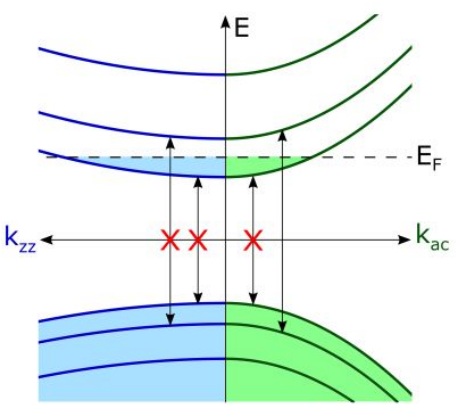

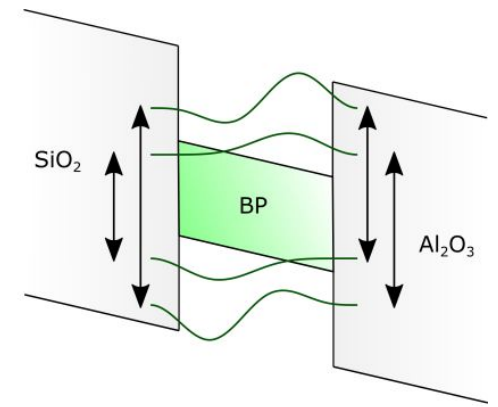

e

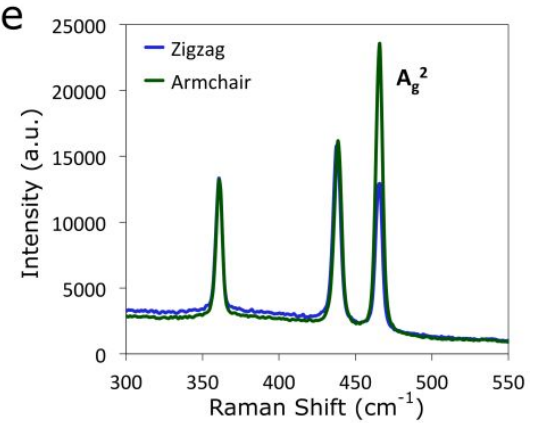

Figure 1. Anisotropic electro-optical effects in few-layer BP. (a) Schematic figure of infrared tunability devices. Few-layer BP is mechanically exfoliated on $285 \mathrm{~nm} \mathrm{SiO} 2 / \mathrm{Si}$ and then capped with $45 \mathrm{~nm} \mathrm{Al} \mathrm{O}_{3}$ by ALD. A semitransparent top contact of $5 \mathrm{~nm} \mathrm{Pd}$ is used to apply field $\left(\mathrm{V}_{\mathrm{G} 1}\right)$ while the device floats and $20 \mathrm{~nm} \mathrm{Ni} / 200 \mathrm{~nm}$ Au contacts are used to gate $\left(V_{\mathrm{G} 2}\right)$ the contacted device. (b) Crystal structure of BP with armchair (AC) and zigzag (ZZ) axes indicated. (c) Illustration of two field-driven electro-optical effects: the quantum-confined Stark effect, and symmetry-breaking modification of quantum well selection rules. In the quantum-confined Stark effect, an external field tilts the quantum well energy levels, causing a red-shifting of the intersubband transition energies. In the observed modification of selection rules, this field breaks the symmetry of the quantum well and orthogonality of its wavefunctions, allowing previously forbidden transitions to occur (eg: from $v_{1}$ to $c_{2}$, a previously disallowed transition). (d) Illustration of anisotropic Pauli-blocking (Burstein-Moss effect) in BP. Intersubband transitions are blocked due to the filling of the conduction band. Along the $Z Z$ axis, all optical transitions are disallowed regardless of carrier concentration. (e) Raman spectra with excitation laser polarized along $A C$ and $Z Z$ axes. The intensity ratio between the $A_{g}{ }^{2}$ peak and the $A_{g}{ }^{1}$ peak is used to identify crystal axes.

To illustrate the mechanisms of tunable dichroism of BP in the mid-infrared, we measure tunability of transmittance using Fourier-Transform Infrared (FTIR) microscopy as a function of externally $\left(\mathrm{V}_{\mathrm{G} 1}\right)$ or directly applied bias $\left(\mathrm{V}_{\mathrm{G} 2}\right)$, presented for a $3.5 \mathrm{~nm}$ thick flake, as determined from atomic force microscopy (AFM) (Figure S1, Supporting Information), in Figure 2. Fig. 2b 
presents the raw extinction of the flake along the armchair axis at zero bias, obtained by normalizing the armchair axis extinction to that of the optically inactive zigzag axis. A band edge of approximately $0.53 \mathrm{eV}$ is measured, consistent with a thickness of $3.5 \mathrm{~nm}$. A broad, weak shoulder feature is observed at approximately $0.75 \mathrm{eV}$. The corresponding calculated optical constants for the flake are presented in Figure $2 c$ for comparison. A Kubo formula approach is used for this calculation, as is described in further detail under Calculations in the Methods section, and in Ref [35-37].
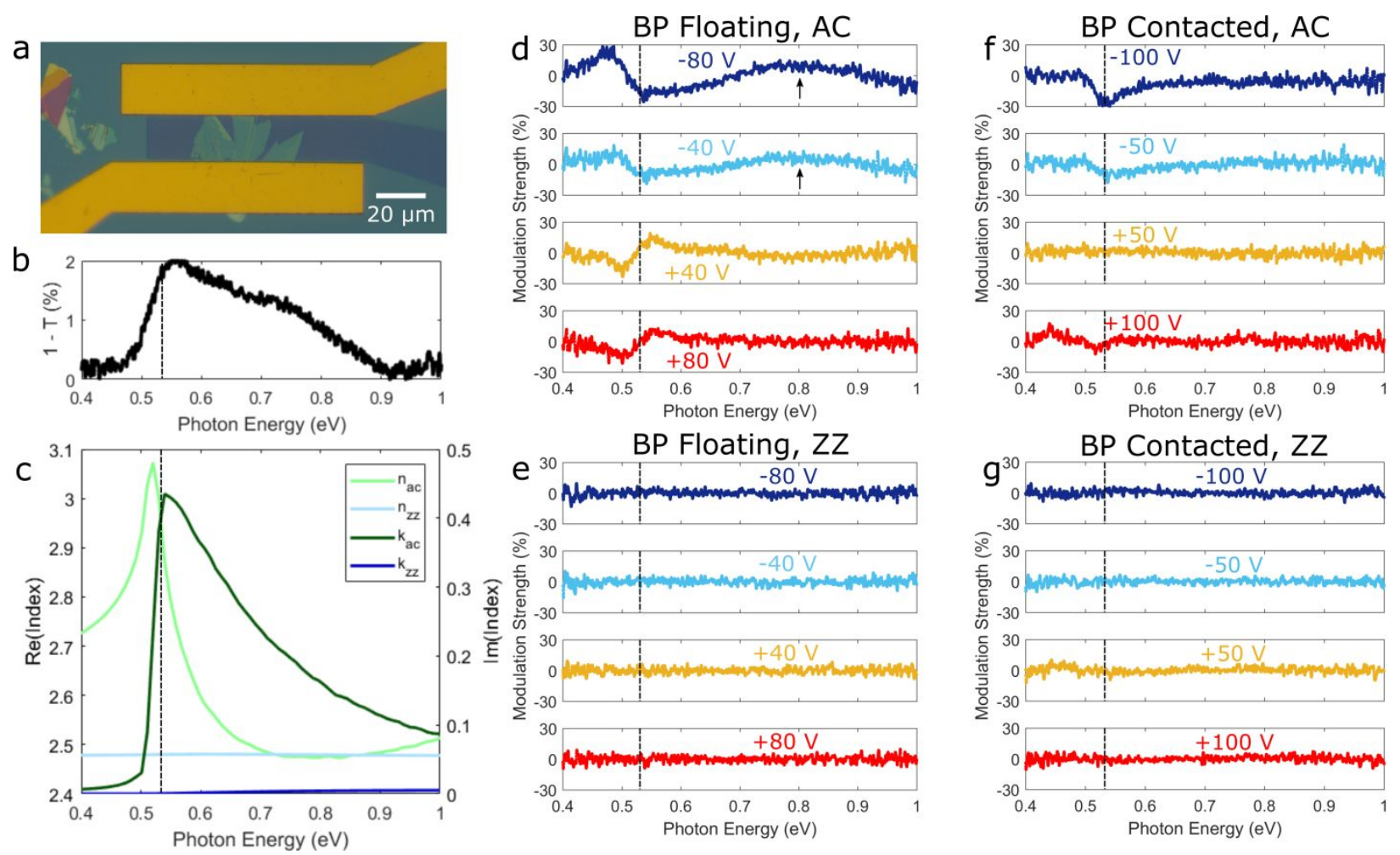

Figure 2. Electrically tunable linear dichroism: quantum-confined Stark and Burstein-Moss effects and forbidden transitions. (a) Optical image of fabricated sample with two Au contact pads for direct electrical contact to the BP and a semi-transparent Pd top contact between them, separated from the BP by a thin layer of $\mathrm{Al}_{2} \mathrm{O}_{3}$, as in the schematic of Figure $1 \mathrm{a}$; (b) Zerobias infrared extinction of $3.5 \mathrm{~nm}$ flake, polarized along armchair (AC) axis. (c) Calculated index of refraction for $3.5 \mathrm{~nm}$ thick BP with a Fermi energy at mid-gap. (d) Tunability of BP oscillator strength with field applied to floating device, for light polarized along the $A C$ axis. (e) Corresponding tunability for light polarized along the zigzag (ZZ) axis. (f) Tunability of BP oscillator strength with gating of contacted device, for light polarized along the AC axis. (g) Corresponding tunability for light polarized along the $\mathrm{ZZ}$ axis. 
Figures $2 d$ and $2 e$ illustrate the influence of an external field on the extinction of BP with carrier concentration held constant (i.e. the BP is left floating). The extinction data for each voltage is normalized to the zero bias case and to the peak BP extinction seen in Figure $2 b$, to obtain a tuning strength percentage that quantifies the observed tunability of the BP oscillator strength. In total, this tuning strength percentage at voltage $V^{\prime}$ is defined as follows.

$$
\begin{gathered}
\text { Modulation Strength }(\%)=100 \times \frac{-\Delta T_{V^{\prime} / 0 V}(\%)}{\max \left(-\Delta T_{A C / Z Z}\right)(\%)} \\
\text { where: }-\Delta T_{A / B}(\%)=100 \times\left(1-\frac{T_{A}}{T_{B}}\right)
\end{gathered}
$$

As an example, for the device examined in Fig. 2, the maximum value of $-\Delta T_{A C / Z Z}$ is shown in Fig. $2 \mathrm{~b}$ to be approximately 2 percent. At its strongest, $-\Delta T_{V^{\prime} / 0 \mathrm{~V}}$ approaches (for $-80 \mathrm{~V}$ applied, and a photon energy of $0.475 \mathrm{eV}$ ) about one third of this 2 percent maximal BP extinction value, yielding a modulation strength of $30 \%$. We note that this normalization scheme underestimates the tuning strength for photon energies where overall BP extinction is lower, such as away from any subband edges. Along the armchair axis, presented in Fig. $2 d$, two tunable features are measured near photon energies of 0.5 and $0.8 \mathrm{eV}$. We explain the first feature at $0.5 \mathrm{eV}$ as arising from a shifting of the BP band edge due to the quantum-confined Stark effect. At negative bias, the band gap effectively shrinks and this is manifest as a redistribution of oscillator strength near the band edge to lower energies. As a result, an increase in absorptance is measured below the zero-bias optical band gap, and a decrease is seen above it. At positive bias, this trend is weakened and reversed. We propose two explanations for this asymmetry: the first is the influence of electrical hysteresis, and the second is the presence of a small internal field in the BP at zero bias, which has been observed in previous works on the infrared optical response of few-layer $\mathrm{BP}^{15}$.

The second, higher energy feature observed in the measured spectrum does not correspond to any predicted intersubband transition. Rather, we propose it arises due to the modification of quantum well selection rules that limit the allowed intersubband optical transitions in black phosphorus by the applied electric field. In a symmetric quantum well, only transitions between states with equal quantum numbers are allowed, as other states have orthogonal 
wavefunctions with zero overlap integrals ${ }^{36}$. However, a strong applied field breaks the symmetry of the quantum well and the orthogonality of its wavefunctions, eliminating this selection rule. We can likely assign this new feature to the first hybrid transition, $E_{12}$, consistent with literature results for a flake of the same thickness (6-layers) and zero-bias band gap energy $^{15}$. Due to some uncertainty in flake thickness, this feature could also be well-explained by a combination of the first two hybrid transitions for a 7-layer sample. We note that this feature is present in the $0 \mathrm{~V}$ extinction spectrum, consistent with a zero-bias internal field. As the symmetry is further broken with an externally-applied electric field, this transition is strengthened. Under positive bias, the internal and external fields are in competition, resulting in minimal change. This suppressed tunability can also be attributed to hysteresis, as before.

In Figure 2e, no tunability is measured for any applied bias for light polarized along the zigzag axis. This can be well understood due to the dependence of the Stark effect on the initial oscillator strength of an optical transition; because no inter-subband optical transitions are allowed along this axis, the field effect is weak. Similar behavior has been observed in excitons in $\mathrm{ReS}_{2}$ based on an optical Stark effect ${ }^{45}$. Moreover, while the externally applied field can allow 'forbidden' transitions along the armchair axis by breaking the out-of-plane symmetry of the quantum well, in-plane symmetry properties and thus the selection rule precluding all zig-zag axis inter-subband transitions are unaffected. This selection rule and the corresponding symmetry properties have been previously described ${ }^{19}$.

In Figures $2 \mathrm{f}$ and $2 \mathrm{~g}$, we present the complementary data set of tunable dichroism measurements due to a directly applied gate bias with electrical contact made to the BP in a standard field-effect transistor (FET) geometry. Here, we observe tunability dominated by carrier concentration effects. At the band gap energy of approximately $0.53 \mathrm{eV}$, a simple decrease in absorptance is observed at negative and large positive biases, consistent with an ambipolar BM shift. Unlike the results of applying field while the BP floats, no tunability of the forbidden transition at $0.75 \mathrm{eV}$ is observed; this is explained in part due to the screening of the electric field due to the carrier concentration tunability. We additionally may consider the 
possibility that this optical transition is disallowed by Pauli-blocking effects, negating the symmetry-breaking effect of the directly applied field. As in the case for the floating BP measurement, no tunability is observed along the zigzag axis.

The anisotropic electro-optical effects described above change character rapidly as the BP thickness - and hence band gap and band structure - is varied. Figure 3 presents analogous results on a flake of $8.5 \mathrm{~nm}$ thickness, determined by AFM (see Supporting Information, Fig. S1), for which an optical image is presented in Fig. 3a. Due to the increased thickness, the energy separation between subbands is smaller, resulting in a narrower free-spectral range between absorptance features measured in the zero-bias spectrum, presented in Fig. $3 \mathrm{~b}$ and for which corresponding calculated optical constants are presented in Fig. 3c. Results for tunability by an external field with the BP left floating are presented in Fig. 3d. As in the thin flake, substantial tuning of the absorptance at each intersubband transition is observed due to the quantumconfined Stark effect (QCSE) red-shifting the energy of the subbands. Due to the large Stark coefficient in BP - which increases with thickness in the few-layer limit - absorption is nearly $100 \%$ suppressed, resulting in an approximately isotropic optical response from the material ${ }^{6,46}$. Unlike the previous sample, tuning of forbidden transitions is not apparent; all features correspond to transitions measured in the $0 \mathrm{~V}$ normalization scheme as well as the calculated optical constants for a thickness of $8.5 \mathrm{~nm}$. As before, no tuning is seen along the zigzag axis, as shown in Figure S2, Supporting Information. In Fig. 3e, the tunability for directly gated, contacted BP is shown. The observed tuning - a reduction in extinction centered at each of the calculated intersubband transition energies - is relatively weak and does not persist to high photon energies. This suggests that the dominant tunability mechanism is the ambipolar BM shift, rather than the QCSE. Additional measurements at lower energies are presented in Figure S3, Supporting Information. 

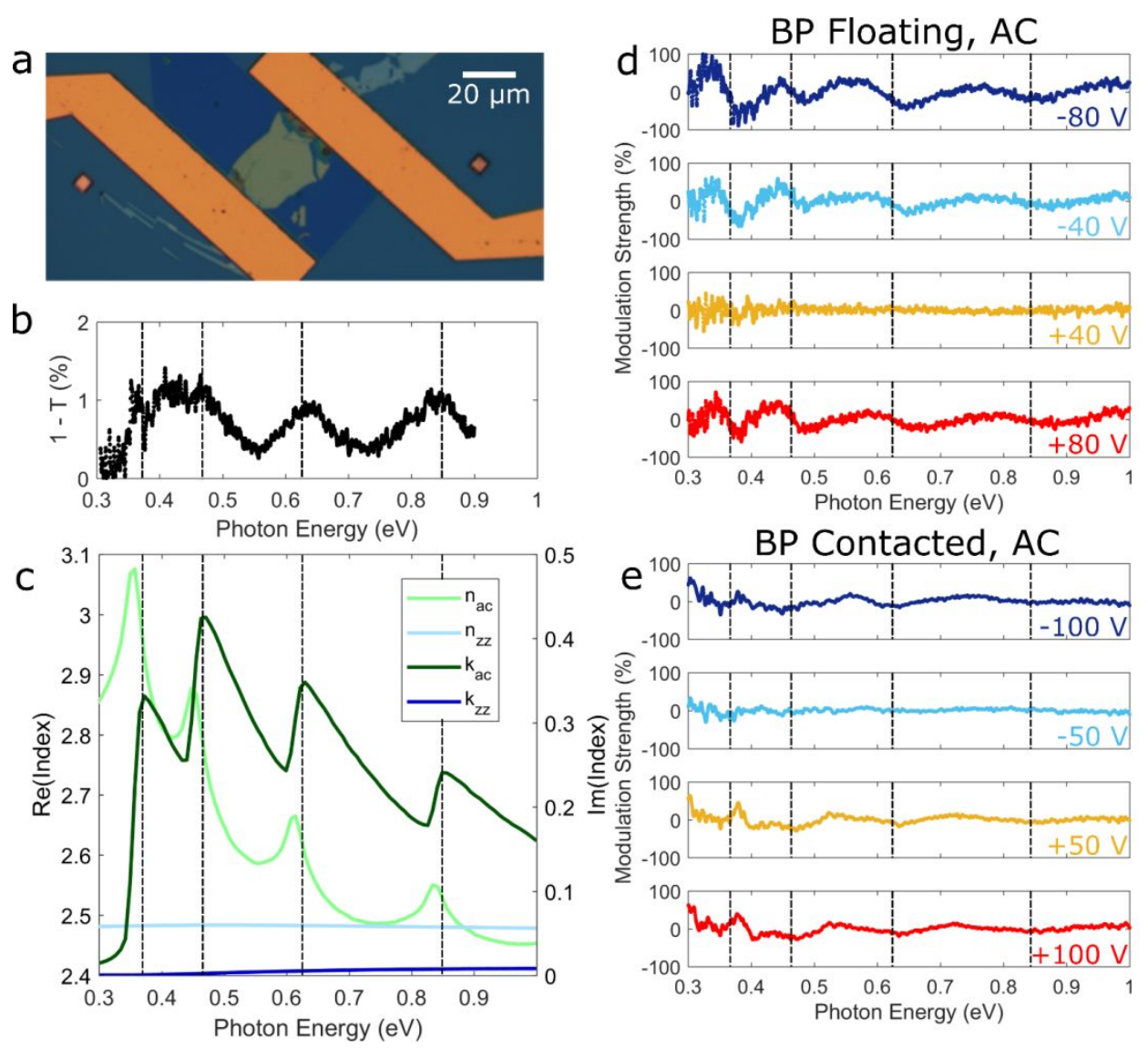

Figure 3. Variation of Tunability with BP Thickness. (a) Optical image of fabricated $8.5 \mathrm{~nm}$ sample. (b) Zero-bias extinction of $8.5 \mathrm{~nm}$ flake, polarized along AC axis. (c) Calculated index of refraction for $8.5 \mathrm{~nm}$ thick BP. (d) Tuning of BP oscillator strength with field applied to floating device, for light polarized along the AC axis. (e) Tuning of BP oscillator strength with gating of contacted device, for light polarized along the AC axis.

In Figure 4 we present results of gate-tunable dichroism at visible frequencies in a $20 \mathrm{~nm}$ thick flake. A new device geometry is used to enable transmission of visible light, shown schematically in Fig. 4a and in an optical image in Fig. 4b. In this configuration, a $\mathrm{SrTiO}_{3}$ substrate is utilized to allow transmission-mode measurements at visible wavelengths. A symmetric gating scheme is devised based on semi-transparent top and back gate electrodes of $5 \mathrm{~nm} \mathrm{Ni}$, as described in the Methods section. Only an applied field, floating BP measurement is utilized, as band-filling effects should be negligible at this energy range. In Fig. 4c, we present tunability results from 1.3 to $2 \mathrm{eV}$. Due to the QCSE, tunability is observed up to $1.8 \mathrm{eV}$, corresponding to red light. Thus we demonstrate that electro-optic tuning of linear dichroism is 
possible across an extraordinarily wide range of wavelengths in a single material system, enabling multifunctional photonic devices with broadband operation.
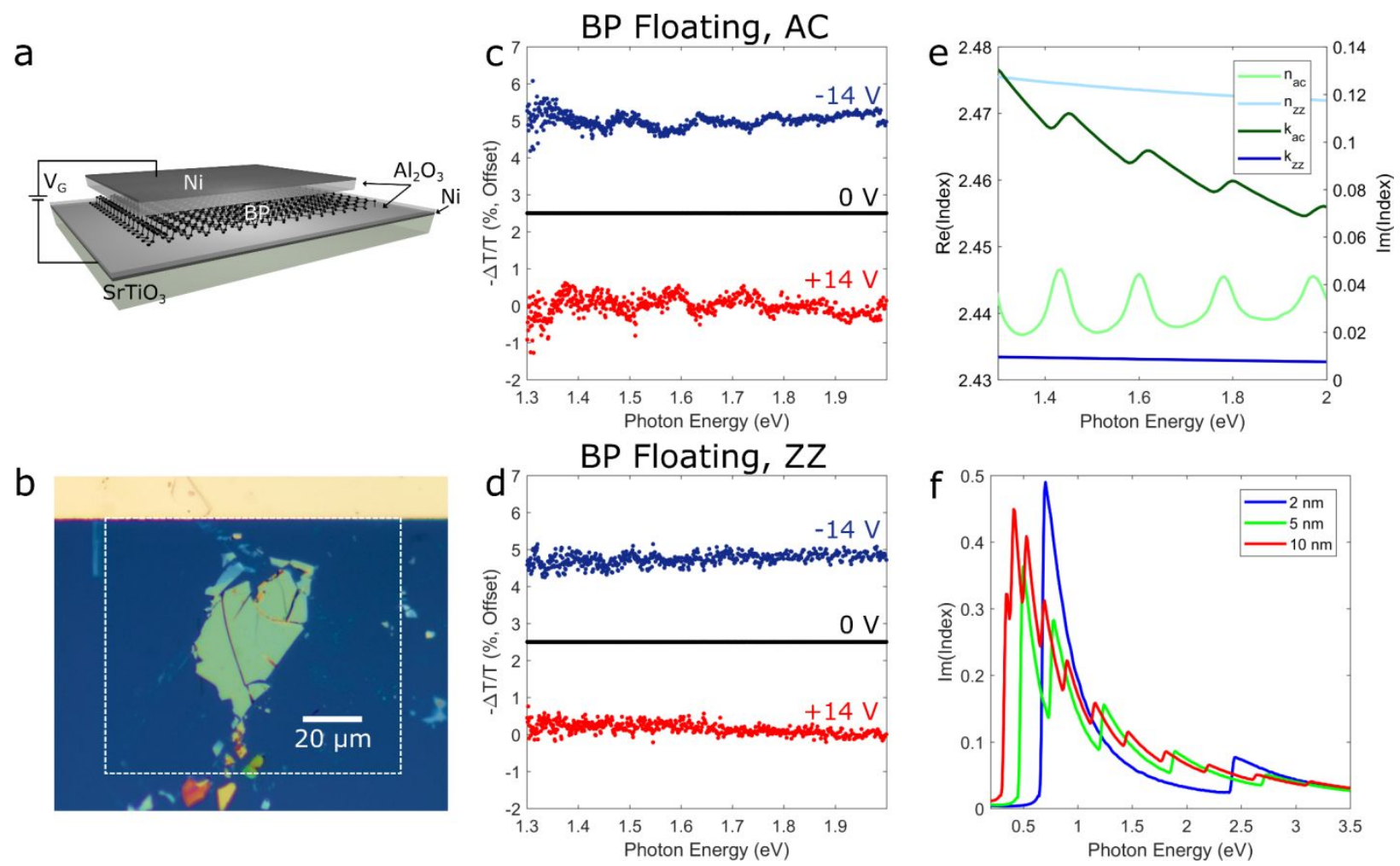

Figure 4. Tunability in the Visible. (a) Schematic figure of visible tuning device. Few-layer BP is mechanically exfoliated on $45 \mathrm{~nm} \mathrm{Al}_{2} \mathrm{O}_{3} / 5 \mathrm{~nm} \mathrm{Ni}$ on $\mathrm{SrTiO}_{3}$ and then coated with $45 \mathrm{~nm} \mathrm{Al}_{2} \mathrm{O}_{3}$. A $5 \mathrm{~nm}$ thick semitransparent Ni top contact is used. (b) Optical image of fabricated sample with $20 \mathrm{~nm}$ thick BP. Dashed white line indicates the boundary of the top Ni contact. (c) Tuning of extinction with field applied to floating device, for light polarized along the AC axis. (d) Corresponding tuning for light polarized along the ZZ axis. (e) Calculated index of refraction for $20 \mathrm{~nm}$ thick BP for the measured energies. (f) Calculated imaginary index of refraction of several thicknesses of BP from the infrared to visible.

The decay of BP intersubband oscillator strength at higher photon energies provides a spectral cutoff for QCSE-based tunability, but for $5 \mathrm{~nm} \mathrm{BP}$ or thinner this oscillator strength is strong through the entire visible regime, as illustrated in Fig. $4 \mathrm{f}$. We thus suggest that in very thin BP, strong tuning of absorption and dichroism is possible to even higher energies. By selecting a flake of $2 \mathrm{~nm}$, for example, tunable linear dichroism is possible up to $3 \mathrm{eV}$ from the band gap energy of $0.75 \mathrm{eV}$. A higher density of features, beginning at lower energies, may be introduced by utilizing a thicker flake, with slightly decreased tuning strength, as seen for 5 and $10 \mathrm{~nm}$ 
thickness flakes. We also note that by substituting graphene top and bottom contacts or utilizing nanophotonic techniques to focus light in the BP, higher absolute tuning strength could be easily realized.

This phenomenon is in stark contrast to the gate-tunability of the optical response of other 2D materials, where substantial tunability is typically constrained to the narrowband energy of the primary exciton, as in $\mathrm{MoS}_{2}$ and $\mathrm{WS}_{2}{ }^{1,47}$. In another van der Waals materials system, monolayer graphene, tunability is accessible over a broader wavelength range due to the Pauli-blocking of optical transitions at $2 \mathrm{E}_{\mathrm{F}}$; however, this is limited to the range over which electrostatic gating is effective, typically between $\mathrm{E}_{\mathrm{F}} \sim 0$ to $\mathrm{E}_{\mathrm{F}} \sim 0.5 \mathrm{eV}^{2,48}$. Moreover, these materials are not dichroic or birefringent in-plane, and so BP offers a novel phenomenon that can be taken advantage of to realize previously challenging or impossible photonic devices. The same restriction is true of bulk tunable materials such as quantum wells, transparent conducting oxides, and transition metal nitrides.

In summary, we have observed and isolated three competing, anistropic electro-optical effects in few-layer black phosphorus: the Pauli-blocking of intersubband transitions (Burstein-Moss effect), the quantum-confined Stark effect, and the modification of quantum well selection rules by a symmetry breaking electric field. These effects, which produce near unity changes in the black phosphorus oscillator strength for some material thicknesses and photon energies, can be tuned to a broad range of frequencies, from the mid infrared to the visible, by controlling the thickness and thus band structure of the black phosphorus. Further, these are strongly anisotropic electro-optical effects, and we thus observe them to allow electrical control of both linear dichroism and birefringence. We observe that absorption in BP can be tuned from anisotropic to nearly isotropic. We suggest that these phenomena and this material are a promising platform for controlling the in-plane propagation of surface or waveguide modes, as well as for polarization-switching, phase and amplitude control, and reconfigurable far-field metasurfaces. As with van der Waals materials as a whole, few layer black phosphorus 
provides a route not only to an improved material platform for optoelectronics, but also to new physics, and the potential new technology paradigms that follow. 


\section{Methods}

Infrared Sample Preparation:

Samples for infrared measurements were fabricated by mechanically exfoliating few-layer BP onto $285 \mathrm{~nm} \mathrm{SiO} / \mathrm{Si}$ in a glove box environment. Contacts of $20 \mathrm{~nm} \mathrm{Ni} / 200 \mathrm{~nm} \mathrm{Au}$ were fabricated by electron beam lithography, electron beam evaporation, and liftoff. A top gate dielectric of $45 \mathrm{~nm} \mathrm{Al}_{2} \mathrm{O}_{3}$ was deposited by atomic layer deposition (ALD) following the technique in Ref. ${ }^{49}$, and a semi-transparent top contact of $5 \mathrm{~nm}$ Ni was deposited by electron beam evaporation and liftoff. Measurements were performed in a Fourier Transform Infrared Spectrometer coupled to a microscope.

Visible Sample Preparation:

Samples for visible measurements were fabricated by depositing a $5 \mathrm{~nm}$ thick semi-transparent back contact of $\mathrm{Ni}$, followed by $45 \mathrm{~nm} \mathrm{Al}{ }_{2} \mathrm{O}_{3}$ by ALD on a $0.5 \mathrm{~mm}$ thick $\mathrm{SrTiO}_{3}$ substrate. Fewlayer BP was then mechanically exfoliated and electrical contacts were fabricated as above. Measurements are performed in a visible spectrometer. Nickel was selected as the optimum metallic contact through Finite-Difference Time Domain simulations presented in Figure S4, Supporting Information.

Calculations:

Calculations of the optical constants of BP are based on the formalism developed in Ref. [36]. Optical conductivity $\sigma$ is calculated using the Kubo formula within an effective low-energy Hamiltonian for different thicknesses. The permittivity is calculated as $\varepsilon(\omega)=\varepsilon_{\infty}+i \sigma / \omega \delta$ where $\delta$ is the thickness of the $B P$, and the high-frequency permittivity $\varepsilon_{\infty}$ is taken from Ref. [41].

\section{Associated Content}

Supporting Information Available: Identification of crystal axes, AFM characterization, additional sample spectra, visible sample substrate simulations, high modulation strength sample spectra, comparison to theory 


\section{Author Information}

Corresponding Author: E-mail: haa@caltech.edu

ORCID

Michelle C. Sherrott: 0000-0002-7503-9714

Harry A. Atwater: 0000-0001-9435-0201

Author Contributions: M.C.S. and W.S.W. are equal contributors.

Notes: The authors declare no competing financial interest.

\section{Acknowledgements:}

The authors gratefully acknowledge support from the Department of Energy, Office of Science under Grant DE-FG02-07ER46405 and for facilities of the DOE "Light-Material Interactions in Energy Conversion" Energy Frontier Research Center (DE-SC0001293). W.S.W. also acknowledges support from an NDSEG Graduate Research Fellowship. M.C.S., D.J. and C.M.W. acknowledge fellowship support from the Resnick Institute. J.W. acknowledges support from the National Science Foundation Graduate Research Fellowship under grant no. 1144469.

\section{References:}

(1) Mak, K. F.; He, K.; Lee, C.; Lee, G. H.; Hone, J.; Heinz, T. F.; Shan, J. Tightly Bound Trions in Monolayer $\mathrm{MoS}_{2}$. Nat. Mater. 2013, 12, 207-211.

(2) Fei, Z.; Rodin, A. S.; Andreev, G. O.; Bao, W.; McLeod, A. S.; Wagner, M.; Zhang, L. M.; Zhao, Z.; Thiemens, M.; Dominguez, G.; et al. Gate-Tuning of Graphene Plasmons Revealed by Infrared Nano-Imaging. Nature 2012, 487, 82-85.

(3) Li, L.; Yu, Y.; Ye, G. J.; Ge, Q.; Ou, X.; Wu, H.; Feng, D.; Chen, X. H.; Zhang, Y. Black Phosphorus Field-Effect Transistors. Nat. Nanotechnol. 2014, 9, 372-377.

(4) Ling, X.; Wang, H.; Huang, S.; Xia, F.; Dresselhaus, M. S. The Renaissance of Black Phosphorus. Proc. Natl. Acad. Sci. 2015, 112, 4523-4530.

(5) Kim, J.; Baik, S. S.; Ryu, S. H.; Sohn, Y.; Park, S.; Park, B.-G.; Denlinger, J.; Yi, Y.; Choi, H. J.; Kim, K. S. Observation of Tunable Band Gap and Anisotropic Dirac Semimetal State in Black Phosphorus. Science 2015, 349, 723-726.

(6) Liu, Y.; Qiu, Z.; Carvalho, A.; Bao, Y.; Xu, H.; Tan, S. J. R.; Liu, W.; Castro Neto, A. H.; Loh, K. P.; Lu, J. Gate-Tunable Giant Stark Effect in Few-Layer Black Phosphorus. Nano Lett. 2017, 17, 1970-1977. 
(7) Deng, B.; Tran, V.; Xie, Y.; Jiang, H.; Li, C.; Guo, Q.; Wang, X.; Tian, H.; Koester, S. J.; Wang, H.; et al. Efficient Electrical Control of Thin-Film Black Phosphorus Bandgap. Nat. Commun. 2017, 14474.

(8) Peng, R.; Khaliji, K.; Youngblood, N.; Grassi, R.; Low, T.; Li, M. Midinfrared Electro-Optic Modulation in Few-Layer Black Phosphorus. Nano Lett. 2017, 17, 6315-6320.

(9) Youngblood, N.; Chen, C.; Koester, S. J.; Li, M. Waveguide-Integrated Black Phosphorus Photodetector with High Responsivity and Low Dark Current. Nat. Photonics 2015, 9, 247-252.

(10) Wang, T.; Hu, S.; Chamlagain, B.; Hong, T.; Zhou, Z.; Weiss, S. M.; Xu, Y.-Q. Visualizing Light Scattering in Silicon Waveguides with Black Phosphorus Photodetectors. Adv. Mater. 2016, 28, 7162-7166.

(11) Chen, C.; Youngblood, N.; Li, M. Study of Black Phosphorus Anisotropy on Silicon Photonic Waveguide. In 2015 Optoelectronics Global Conference (OGC); 2015; pp 1-3.

(12) Huang, M.; Wang, M.; Chen, C.; Ma, Z.; Li, X.; Han, J.; Wu, Y. Broadband BlackPhosphorus Photodetectors with High Responsivity. Adv. Mater. 2016, 28 (18), 3481-3485.

(13) Chen, C.; Youngblood, N.; Peng, R.; Yoo, D.; Mohr, D. A.; Johnson, T. W.; Oh, S.-H.; Li, M. Three-Dimensional Integration of Black Phosphorus Photodetector with Silicon Photonics and Nanoplasmonics. Nano Lett. 2017, 17, 985-991.

(14) Wang, X.; Jones, A. M.; Seyler, K. L.; Tran, V.; Jia, Y.; Zhao, H.; Wang, H.; Yang, L.; Xu, X.; Xia, F. Highly Anisotropic and Robust Excitons in Monolayer Black Phosphorus. Nat. Nanotechnol. 2015, 10, 517-521.

(15) Zhang, G.; Huang, S.; Chaves, A.; Song, C.; Özçelik, V. O.; Low, T.; Yan, H. Infrared Fingerprints of Few-Layer Black Phosphorus. Nat. Commun. 2017, 8, 14071.

(16) Liu, Z.; Aydin, K. Localized Surface Plasmons in Nanostructured Monolayer Black Phosphorus. Nano Lett. 2016, 16, 3457-3462.

(17) Wei, Q.; Peng, X. Superior Mechanical Flexibility of Phosphorene and Few-Layer Black Phosphorus. Appl. Phys. Lett. 2014, 104, 251915.

(18) Luo, Z.; Maassen, J.; Deng, Y.; Du, Y.; Garrelts, R. P.; Lundstrom, M. S.; Ye, P. D.; Xu, X. Anisotropic In-Plane Thermal Conductivity Observed in Few-Layer Black Phosphorus. Nat. Commun. 2015, 9572.

(19) Yuan, H.; Liu, X.; Afshinmanesh, F.; Li, W.; Xu, G.; Sun, J.; Lian, B.; Curto, A. G.; Ye, G.; Hikita, Y.; et al. Polarization-Sensitive Broadband Photodetector Using a Black Phosphorus Vertical p-n Junction. Nat. Nanotechnol. 2015, 10, 707-713.

(20) Liao, B.; Zhao, H.; Najafi, E.; Yan, X.; Tian, H.; Tice, J.; Minnich, A. J.; Wang, H.; Zewail, A. H. Spatial-Temporal Imaging of Anisotropic Photocarrier Dynamics in Black Phosphorus. Nano Lett. 2017, 17, 3675-3680.

(21) Xia, F.; Wang, H.; Jia, Y. Rediscovering Black Phosphorus as an Anisotropic Layered Material for Optoelectronics and Electronics. Nat. Commun. 2014, 5458.

(22) Lan, S.; Rodrigues, S.; Kang, L.; Cai, W. Visualizing Optical Phase Anisotropy in Black Phosphorus. ACS Photonics 2016, 3, 1176-1181.

(23) Qiao, J.; Kong, X.; Hu, Z.-X.; Yang, F.; Ji, W. High-Mobility Transport Anisotropy and Linear Dichroism in Few-Layer Black Phosphorus. Nat. Commun. 2014, 5475.

(24) Sherrott, M. C.; Hon, P. W. C.; Fountaine, K. T.; Garcia, J. C.; Ponti, S. M.; Brar, V. W.; Sweatlock, L. A.; Atwater, H. A. Experimental Demonstration of $>230^{\circ}$ Phase 
Modulation in Gate-Tunable Graphene-Gold Reconfigurable Mid-Infrared Metasurfaces. Nano Lett. 2017, 17, 3027-3034.

(25) Park, J.; Kang, J.-H.; Kim, S. J.; Liu, X.; Brongersma, M. L. Dynamic Reflection Phase and Polarization Control in Metasurfaces. Nano Lett. 2017, 17, 407-413.

(26) Huang, Y.-W.; Lee, H. W. H.; Sokhoyan, R.; Pala, R. A.; Thyagarajan, K.; Han, S.; Tsai, D. P.; Atwater, H. A. Gate-Tunable Conducting Oxide Metasurfaces. Nano Lett. 2016, 16, 5319-5325.

(27) Xu, Q.; Schmidt, B.; Pradhan, S.; Lipson, M. Micrometre-Scale Silicon Electro-Optic Modulator. Nature 2005, 435, 325-327.

(28) Kuo, Y.-H.; Lee, Y. K.; Ge, Y.; Ren, S.; Roth, J. E.; Kamins, T. I.; Miller, D. A. B.; Harris, J. S. Strong Quantum-Confined Stark Effect in Germanium Quantum-Well Structures on Silicon. Nature 2005, 437, 1334-1336.

(29) Inoue, T.; Zoysa, M. D.; Asano, T.; Noda, S. Realization of Dynamic Thermal Emission Control. Nat. Mater. 2014, 13, 928-931.

(30) Coppens, Z. J.; Valentine, J. G. Spatial and Temporal Modulation of Thermal Emission. Adv. Mater. 201701275.

(31) Brar, V. W.; Sherrott, M. C.; Jang, M. S.; Kim, S.; Kim, L.; Choi, M.; Sweatlock, L. A.; Atwater, H. A. Electronic Modulation of Infrared Radiation in Graphene Plasmonic Resonators. Nat. Commun. 2015, 7032.

(32) Rodin, A. S.; Carvalho, A.; Castro Neto, A. H. Strain-Induced Gap Modification in Black Phosphorus. Phys. Rev. Lett. 2014, 112, 176801.

(33) Wang, X.; Tian, H.; Xie, W.; Shu, Y.; Mi, W.-T.; Ali Mohammad, M.; Xie, Q.-Y.; Yang, Y.; $\mathrm{Xu}$, J.-B.; Ren, T.-L. Observation of a Giant Two-Dimensional Band-Piezoelectric Effect on Biaxial-Strained Graphene. Npg Asia Mater. 2015, e154.

(34) Xu, K.; Wang, K.; Zhao, W.; Bao, W.; Liu, E.; Ren, Y.; Wang, M.; Fu, Y.; Zeng, J.; Li, Z.; et al. The Positive Piezoconductive Effect in Graphene. Nat. Commun. 2015, 8119.

(35) Whitney, W. S.; Sherrott, M. C.; Jariwala, D.; Lin, W.-H.; Bechtel, H. A.; Rossman, G. R.; Atwater, H. A. Field Effect Optoelectronic Modulation of Quantum-Confined Carriers in Black Phosphorus. Nano Lett. 2017, 17, 78-84.

(36) Low, T.; Rodin, A. S.; Carvalho, A.; Jiang, Y.; Wang, H.; Xia, F.; Castro Neto, A. H. Tunable Optical Properties of Multilayer Black Phosphorus Thin Films. Phys. Rev. B 2014, 90, 075434.

(37) Lin, C.; Grassi, R.; Low, T.; Helmy, A. S. Multilayer Black Phosphorus as a Versatile Mid-Infrared Electro-Optic Material. Nano Lett. 2016, 16, 1683-1689.

(38) Deng, B.; Tran, V.; Xie, Y.; Jiang, H.; Li, C.; Guo, Q.; Wang, X.; Tian, H.; Koester, S. J.; Wang, H.; et al. Efficient Electrical Control of Thin-Film Black Phosphorus Bandgap. Nat. Commun. 2017, 14474.

(39) Burstein, E. Anomalous Optical Absorption Limit in InSb. Phys. Rev. 1954, 93, 632633.

(40) Moss, T. S. Theory of the Spectral Distribution of Recombination Radiation from InSb. Proc. Phys. Soc. Sect. B 1957, 70, 247.

(41) Wang, X.; Lan, S. Optical Properties of Black Phosphorus. Adv. Opt. Photonics 2016, 8, 618-655.

(42) Miller, D. A. B.; Chemla, D. S.; Damen, T. C.; Gossard, A. C.; Wiegmann, W.; Wood, T. H.; Burrus, C. A. Band-Edge Electroabsorption in Quantum Well Structures: The Quantum-Confined Stark Effect. Phys. Rev. Lett. 1984, 53, 2173-2176. 
(43) Ling, X.; Huang, S.; Hasdeo, E. H.; Liang, L.; Parkin, W. M.; Tatsumi, Y.; Nugraha, A. R. T.; Puretzky, A. A.; Das, P. M.; Sumpter, B. G.; et al. Anisotropic Electron-Photon and Electron-Phonon Interactions in Black Phosphorus. Nano Lett. 2016, 16, 2260-2267.

(44) Hsiao, Y.; Chang, P.-Y.; Fan, K.-L.; Hsu, N.-C.; Lee, S.-C. Black Phosphorus with a Unique Rectangular Shape and Its Anisotropic Properties. AIP Adv. 2018, 8, 105216.

(45) Sim, S.; Lee, D.; Noh, M.; Cha, S.; Soh, C. H.; Sung, J. H.; Jo, M.-H.; Choi, H. Selectively Tunable Optical Stark Effect of Anisotropic Excitons in Atomically Thin $\mathrm{ReS}_{2}$. Nat. Commun. 2016, 13569.

(46) Dolui, K.; Quek, S. Y. Quantum-Confinement and Structural Anisotropy Result in Electrically-Tunable Dirac Cone in Few-Layer Black Phosphorous. Sci. Rep. 2015, 11699.

(47) Chernikov, A.; van der Zande, A. M.; Hill, H. M.; Rigosi, A. F.; Velauthapillai, A.; Hone, J.; Heinz, T. F. Electrical Tuning of Exciton Binding Energies in Monolayer $\mathrm{WS}_{2}$. Phys. Rev. Lett. 2015, 115, 126802.

(48) Brar, V. W.; Jang, M. S.; Sherrott, M.; Lopez, J. J.; Atwater, H. A. Highly Confined Tunable Mid-Infrared Plasmonics in Graphene Nanoresonators. Nano Lett. 2013, 13, 2541-2547.

(49) Wood, J. D.; Wells, S. A.; Jariwala, D.; Chen, K.-S.; Cho, E.; Sangwan, V. K.; Liu, X.; Lauhon, L. J.; Marks, T. J.; Hersam, M. C. Effective Passivation of Exfoliated Black Phosphorus Transistors against Ambient Degradation. Nano Lett. 2014, 14, 69646970. 


\section{TOC Graphic:}
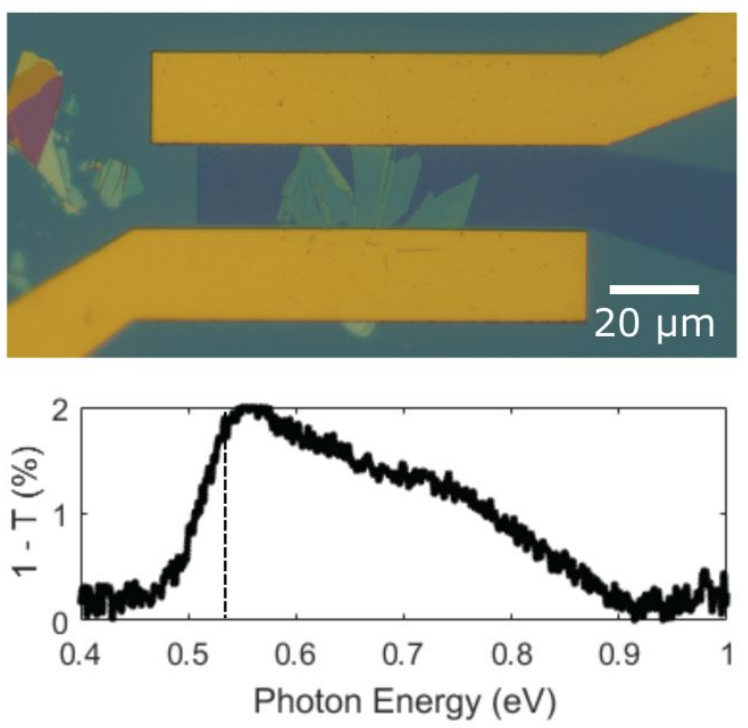

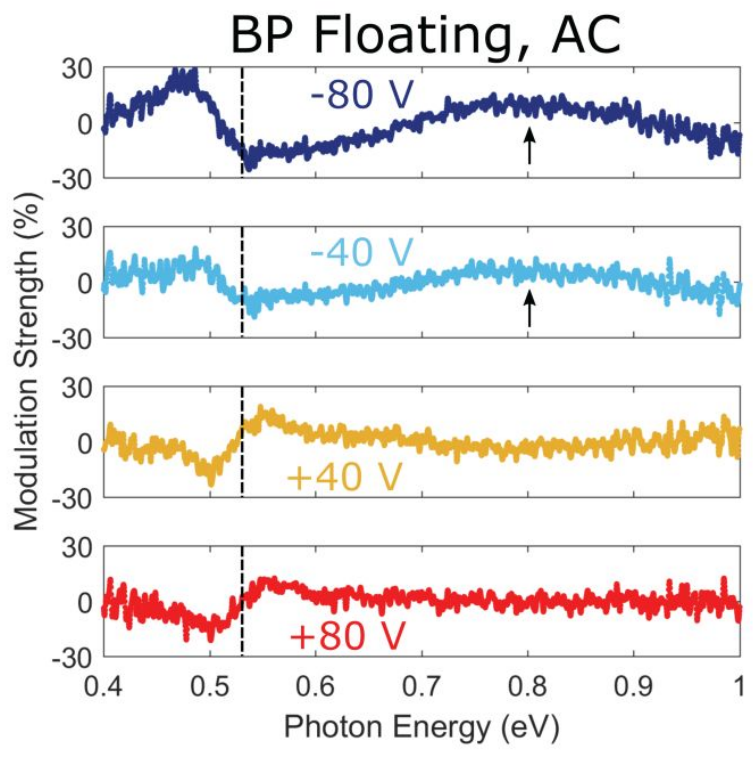

